\title{
Escala de Atitudes em Saúde e Estética: CONSTRUÇÃO E VALIDAÇÃO
}

\author{
Everley Rosane Goetz ; Brigido Vizeu Camargo \\ Universidade Federal de Santa Catarina, Florianópolis, SC, Brasil
}

\section{RESUMO}

O objetivo principal deste estudo foi mensurar atitudes de universitários em relação à saúde e à estética corporais. Utilizou-se a Teoria das Representações Sociais, na abordagem dimensional. Para tanto, foi construida e validada uma Escala de Atitudes em Saúde e Estética - EASE. Participaram 500 universitários dos cursos de Educação Física, Medicina, Nutrição e Moda de Santa Catarina. Observou-se que os estudantes têm atitude levemente positiva frente à saúde e à estética do corpo. Conclui-se que a EASE consiste num instrumento válido para mensuração dessas atitudes, podendo complementar avaliações de pacientes com transtornos dismórficos corporais e obesidade.

Palavras-chave: escala; corpo; atitudes; saúde; estética.

\section{Attitude Scale Health and Beauty: CONSTRUCTION AND VALIDATION}

\begin{abstract}
The main objective of this study was to measure attitudes of university students in relation to health and body aesthetic. The theoretical basis was the Theory of Social Representations, in its dimensional approach. We built the Health and Beauty Attitude Scale - HBAS (construction and validation). 500 university students of Physical Education, Medicine, Nutrition, Fashion of Santa Catarina participated. These students have a slightly positive attitude towards health and body aesthetics. It is concluded that scale is a valid instrument, and may be useful as a complementary tool in evaluation of patients with body dysmorphic disorder and obesity.
\end{abstract}

Keywords: scale; body; attitudes; health; aesthetic.

\footnotetext{
^Endereço para correspondência: Universidade Federal de Santa Catarina, Centro de Filosofia e Ciências Humanas, Departamento de Psicologia. Campus Universitário - Trindade. 88040-900 Florianópolis, SC - Brasil.E-mail: evegoetz@terra.com.br, brigido.camargo@yahoo.com.br
} 
O conceito de corpo pode ser muito amplo e complexo por se relacionar a sentidos diversos, que perpassam aspectos objetivos e subjetivos. Desde a aparência e a saúde física, até aspectos inerentes ao imaginário e à intrasubjetividade do sujeito. As perspectivas e abordagens para a conceituação desse objeto também tangenciam inúmeras áreas e distintas correntes de pensamento, das consideradas clássicas até as atuais (ANDRIEU, 2006; CONTARELLO; FORTUNATI, 2006; GOLDENBERG, 2005; JODELET, 1983, 1994, 2001; JODELET et al., 1982; KNAPP; HALL, 1999; NÓBREGA, 2001; NOVAES; VILHENA, 2003; QUEIROZ, 2000; SERRA; SANTOS, 2003; SOUZA, 2004; STENZEL; GUARESCHI, 2002; WEIL; TOMPAKOW, 1999). Todavia, utilizou-se para este estudo, uma conceituação de corpo que se insere no campo das Ciências Humanas e Sociais, proposta por Andrieu (2006).

Para esse autor, o corpo se caracteriza por uma constituição física que se desenvolve a partir de um programa genético, determinado por variáveis que são definidas pela plasticidade biocultural do sujeito. O corpo também é resultado da interação da matéria genética com o ambiente sociocultural no qual se está inserido, elementos estes que são combinados à medida que se dá a constituição, as regulações e as adaptações. Limita-se à morte, assim como ao mundo exterior e às representações do mundo. Andrieu (2006) acrescenta que os corpos se tornam humanos à medida que assimilam aprendizagens, voluntárias e involuntárias, ao longo de toda a existência humana. Assim, constituem-se de hábitos que vão sendo impressos pela assimilação de códigos, símbolos e linguagens culturais que são peculiares àqueles que os educam em suas vidas.

Enquanto objeto de estudo, que não se limita nem inteiramente ao individual, nem estritamente ao social, o corpo se constitui por meio de uma construção simbólica e de uma invenção subjetiva. Estas vão sendo determinadas pelas percepções e pelas representações individuais e coletivas. Desses aspectos simbólicos e subjetivos, faz parte também a diferenciação sexual, que tem determinantes sociais relacionados à capacidade de reprodução e à organização econômica, norteadas pelas normas de trabalho e da vida cotidiana (ANDRIEU, 2006; SOUZA, 2004).

A saúde é outro aspecto relevante quando se considera a conceituação de corpo. Straub (2005) conceitua saúde como um estado de completo bem-estar físico, mental e social. Para este autor, a saúde não está restrita à mera ausência de doença ou de enfermidade, mas a um estado positivo e multidimensional que envolve três principais domínios, que contemplam aspectos físicos, psicológicos e sociais. Czeresnia e Freitas (2004) acrescentam que a saúde seria a qualidade de vida resultante de um processo complexo determinado por diversos fatores, tais como, alimentação, ecossistema, renda, educação, justiça social, dentre outros. No Brasil, a conceituação de saúde abrange e prevê o direito de condições dignas de vida e de acesso universal às ações e aos serviços de promoção, proteção e recuperação da saúde, de forma igualitária a todos os habitantes do território nacional (BRASIL, 2001). 
Czeresnia e Freitas (2004, p. 42) ponderam ainda que "a saúde e o adoecer são formas pelas quais a vida se manifesta, correspondentes a experiências singulares e subjetivas, impossíveis de serem reconhecidas e significadas integralmente pela palavra". A respeito dessa singularidade e subjetividade, Soares Neto (2006) complementa que qualquer organismo pode ser considerado como estando saudável quando funciona bem, sendo tal condição relacionada à ideia de equilíbrio, vigor, felicidade, autorrealização, tanto em aspectos físicos quanto psíquicos.

A estética é outro foco de relevância científica no atual contexto, que merece destaque para o estudo do corpo. O termo "estética" pode ser comumente atribuído a diversos sentidos em seu uso cotidiano, aplica-se também a diferentes conceitos científicos, inseridos em áreas igualmente distintas: Filosofia, Ciências Humanas, Sociais e da Saúde, dentre outras (ANDRIEU, 2006; BRAUNER; GOMES; LOPES, 2007; CLARKE; GRIFFIN, 2009; HUBERT; DE LABARRE, 2005; KNAPP; HALL; 1999; MANER et al., 2003; QUEIROZ, 2000; QINGXING, XUE; JIAYE, 2000; TEIXEIRA, 2001; TURNER, 2002). Dentre as inúmeras variações do conceito de estética que perpassam do material ao abstrato, do real ao imaginário, do objetivo ao subjetivo, do animado ao inanimado até a mera percepção do que é belo, adotou-se, para esta pesquisa, o termo "estética" enquanto sinônimo de beleza física, como um fenômeno inerente e de relevância ao estudo do corpo.

Isso justificado, porque nas atuais pesquisas sobre o corpo, tem-se observado com frequência estudos relacionando saúde com estética, assim como o surgimento e a descrição de novos problemas relacionando esses dois aspectos inerentes a tal objeto (CALAF et al., 2005; CAMARGO; GOETZ; BARBARÁ, 2005a, 2005b; CAMARGO; GOETZ; JUSTO, 2007; MALYSSE, 2002; TAVARES, 2003; GOETZ, 2013). Especula-se que os problemas associados ao binômio "saúde-estética" sejam um fenômeno crescente na sociedade atual, justificado em parte pelo paradoxo das mensagens difundidas pela mídia (HUBERT; DE LABARRE, 2005), que gera ambiguidades nas percepções, sentimentos, cognições e ações dos indivíduos.

Este paradoxo pode ser constatado pelo aumento dos transtornos dismórficos corporais amplamente difundidos pela mídia, conhecidos pelo senso comum, e investigados cientificamente, como a anorexia e a bulimia (ABREU; CANGELLI FILHO, 2004; CAMBRAIA, 2004; ESPÍNDOLA; BLAY, 2006, 2009; LATTERZA et al., 2004; MARTINS; SASSI JÚNIOR, 2004; MORGAN, 2005; NIEMEYER; KRUSE, 2008; PINZON; NOGUEIRA, 2004; SALZANO; CORDÁS, 2004a, 2004b; SERRA; SANTOS, 2003; SOUTO; FERRO-BUCHER, 2006; WEINBERG; CORDÁS; MUNOZ, 2005); aos atuais, pouco difundidos, mas que também se tornam mais frequentes como a vigorexia, um subtipo do transtorno dismórfico corporal (TDC) que ocorre principalmente em homens que, apesar da grande hipertrofia muscular, consideram-se pequenos e fracos (ASSUNÇÃO, 2002). E, a plasticomania, termo criado e descrito pela autora deste artigo para definir a prática compulsiva por plásticas, que leva muitas pessoas, principalmente mulheres jovens, a insistir em intervenções cirúrgicas recorrentes somente para fins estéticos, em busca da imagem corporal ideal, sem obtenção da devida satisfação (GOETZ, 2009).Em síntese, a busca pela saúde e pela beleza tem alcançado 
notoriedade nos últimos tempos, em especial pela difusão maciça da mídia, que pode ser percebida por meio de diferentes meios de comunicação de massa. Por exemplo, nas capas de revistas comumente se observam contradições, pois, ora enfatizam informações inerentes aos segredos para alcançar a beleza, ora alertam aos problemas de saúde que tal procura pode suscitar (GOETZ et al., 2008).

Outra ambiguidade verificada nitidamente é que frequentemente os programas de televisão, criticam a obesidade, responsabilizando os indivíduos pelo aumento e descontrole de peso; mas, em contrapartida, também divulgam as delícias dos fast-foods, estimulando-os ao consumo de tais produtos (GOETZ, 2009).

Em um estudo realizado sobre as representações sociais, do qual participaram 500 universitários de uma instituição da cidade de Florianópolis, observou-se que o pensamento social compartilhado por quase metade desses jovens participantes é indicativo de uma tendência à modificação do corpo pela intervenção plástico-cirúrgica. Os resultados encontrados foram de que aproximadamente $46 \%$ deles já realizaram ou ainda pretendem realizar algum tipo de cirurgia plástica somente para fins estéticos. Estes incluem a implantação de próteses, enxertos, preenchimentos, reduções e correções de todo tipo para atingirem o padrão corporal considerado ideal. A maior prevalência é de mulheres que pretendem ou já realizaram algum tipo de cirurgia estética, mas os homens também se preocupam com o corpo e a aparência deste. Dentre as mulheres participantes, justificam em relação ao corpo, que pretendem se sentir bonitas quando se comparam a outras mulheres; enquanto que os homens dessa pesquisa centraram a preocupação estética relativa ao corpo no poder de atração (GOETZ, 2009).

Há uma busca incessante pela beleza e pela juventude, que não se restringe apenas a modelos ou candidatas de concursos de beleza, mas também atinge pessoas comuns. Beleza física comumente se torna objeto de desejo e se associa ao poder. Pelos avanços da medicina estética, surgimento de novos produtos, técnicas e tecnologias, a beleza passa a ser vista como um "produto", possível de ser adquirido, em especial quando se detém razoável poder aquisitivo. No sentido oposto, o corpo passa a ser motivo de insatisfação e de inadequação para quem não tem acesso a tais recursos (CAMARGO et al., 2011).

Em evidência pelos meios de comunicação como um produto, o corpo é associado a um padrão estético ideal, que obedece a um modelo rígido e com "medidas precisas". Esse modelo ideal comumente enfatiza o magro, jovem e branco. Muitos sujeitos, principalmente os mais jovens, buscam atingir padrões ideais associados ao corpo, mudando hábitos ou fazendo sacrifícios que podem levá-los à doença e à morte. Isso configura uma espécie de "indústria" do corpo que oferece soluções milagrosas e rápidas, pela indicação de medicamentos, cirurgias, implantes e próteses, que são em geral desnecessários ou prejudiciais. No sentido inverso, a obesidade torna-se objeto de medo e até de discriminação, estando, por vezes, associada à falta de cuidado do sujeito e até de sucesso (CAMARGO et al., 2011).

Os meios de comunicação de massa são determinantes nessa busca pela beleza e estimulam pela difusão maciça a "fabricação" de tais padrões corporais. Há uma crescente comercialização do corpo, que é visível na cultura atual e de- 
termina um paradoxo entre a beleza natural e a artificial. Tal paradoxo se associa à insatisfação das pessoas com a própria imagem corporal e contribui decisivamente para o aumento de transtornos do comportamento associados à estética, em especial os Transtornos Dismórficos Corporais (TDCs), tais como a anorexia, a bulimia, a vigorexia e a plasticomania (GOETZ, 2009).

Outro aspecto a ser considerado consiste na diversidade corporal apresentada pela população, que acaba legitimando o surgimento da insatisfação em relação à imagem, podendo ocorrer mesmo quando o corpo está adequado aos padrões de saúde (HUBERT; DE LABARRE, 2005). Sobre isso, há evidente ambiguidade nas difusões midiáticas, que ao mesmo tempo em que estimulam o corpo magro e belo (obtido a partir de todo e qualquer sacrifício), também alertam para os riscos do abuso de substâncias associadas à busca do corpo ideal e dos transtornos alimentares, pela divulgação constante de casos de morte de jovens por anorexia, bulimia e vigorexia.

A obesidade é outro fenômeno crescente da atualidade, que consiste em um conjunto de sinais e de sintomas caracterizado pelo acúmulo excessivo de gordura corporal, e associada ao risco para o desenvolvimento de doenças crônicas. Abrange tanto aspectos de saúde quanto de estética e cresce como uma epidemia, gerando insatisfação e a busca por soluções, dentre elas, cresce o número de pessoas que buscam cirurgias bariátricas, em especial nos casos em que há riscos de morbidade (BUENO; FISBERG, 2006; LORDELO et al., 2007; LOTUFO, 2006).

Camargo (2003) ressalta o papel da mídia como determinante na formação das representações sociais e das atitudes das pessoas. Hubert e De Labarre (2005) acrescentam a isso, a relevância da mídia principalmente na formação das representações e atitudes relacionadas a aspectos corporais. Camargo et al. (2011) destacam que as representações sobre o corpo, socialmente difundidas na mídia impressa, evidenciam modelos e padrões de beleza - com ênfase no corpo remodelado, produzido, jovem e "tecnológico", como saber compartilhado pelo senso comum.

As representações sociais são formas pelas quais se constitui o conhecimento do mundo, pelo agrupamento de conjuntos de significados que permitem dar sentido aos fenômenos ou fatos novos, que formam um saber compartilhado. Esse saber assume certa generalidade e funcionalidade para as pessoas, constituindo o senso comum. Portanto, as representações sociais são um conjunto de conhecimentos, explicações e afirmações, que são originadas no cotidiano dos sujeitos, pelo decurso das comunicações interindividuais (JODELET, 1986, 2001; MOSCOVICI, 1978, 2003).

Como integrantes de uma das dimensões do campo representacional, as atitudes impregnam as representações sociais e estas últimas são determinantes nas condutas desejáveis ou esperadas socialmente (VALA; MONTEIRO, 1996). Segundo Rodrigues, Assmar e Jablonski (2002) e Ajzen (2001), as atitudes são definidas como experiências subjetivas, que incluem sempre uma dimensão avaliativa em relação a um objeto, um fato ou uma pessoa. 
Rodrigues, Assmar e Jablonski (2002) consideram ainda, que as atitudes envolvem o que as pessoas pensam, sentem e como elas gostariam de se comportar frente a um objeto ou fenômeno. Ou seja, o comportamento não é determinado somente pelo que as pessoas gostariam de fazer, mas também pelo que pensam que devem fazer, ou seja, pelas normas sociais, pelos hábitos e pelo que consideram que é esperado socialmente.

Diante dessas constatações, com o intuito de investigar atitudes de estudantes universitários sobre o corpo, contemplando problemas de relevância sobre o binômio "saúde-estética", foi elaborada uma escala para mensurar comportamentos, hábitos, cuidados e opiniões relativas ao corpo, cujos processos de construção e validação são descritos a seguir.

\section{Método}

A partir da síntese das representações sociais difundidas na mídia impressa, contendo afirmações mais frequentes, expressivas e polêmicas sobre o corpo, coletadas de artigos de revistas nacionais em um estudo prévio (GOETZ et al., 2008), e das representações sociais de universitários sobre saúde e estética obtidas a partir de outro estudo anterior a este (CAMARGO et al., 2011), foi elaborada uma escala para mensurar as atitudes dos participantes a respeito da saúde e da estética corporal, a Escala de Atitudes em Saúde e Estética - EASE.

\section{Participantes}

Os participantes do estudo foram 500 alunos regularmente matriculados nos cursos de Graduação em Educação Física, Nutrição, Medicina e Moda, sendo os três primeiros cursos pertencentes à rede de ensino superior federal de Santa Catarina e o último, à estadual de Santa Catarina, que responderam à EASE. A escolha por esses cursos foi justificada por se considerar que em cada um deles há algum tipo de enfoque predominante no estudo do corpo, seja em aspectos de saúde ou de estética. Além disso, pressupõe-se que os universitários constituam um grupo diferenciado no que diz respeito a ter acesso ao saber científico, cujas atitudes e representações sociais constituem foco de interesse nesta pesquisa.

Destes 500 universitários, 100 participaram do Estudo-Piloto e 400 do Estudo Final, sendo $71,5 \%$ deles do sexo feminino, cuja média de idade foi de 21 anos e 7 meses ( $\mathrm{DP}=4,89)$, sendo a mínima 16 anos e 11 meses e a máxima 56 anos. A idade que apareceu mais frequentemente foi 20 anos e 09 meses, e o valor mais intermediário foi 21 anos. Os estudantes estavam em diferentes períodos de estudo, variando entre o $3^{\circ}$ e o $10^{\circ}$ semestres. A média de tempo de aplicação do instrumento final foi de 10 minutos.

\section{Instrumentos}

Para a construção da escala foram selecionados 60 itens, conforme sugerem Alchieri e Cruz (2003), considerando as fases de construção e validação, que pressupõem descarte dos inadequados (validade de face e de construto), para que a escala final contenha um número aproximado a 20 itens (PASQUALI, 1999). As 
medidas escalares são as mais utilizadas em Psicologia Social, especificamente quando se pretende estudar atitudes (PASQUALI, 1999). A escala definida para este estudo foi do tipo Likert, sendo que cada uma das afirmações (itens) conteve cinco opções de resposta com termos que variaram entre: "muito favorável", "favorável", "nem favorável, nem desfavorável", "pouco favorável" e "desfavorável". A pontuação desses termos varia de cinco a um, se a resposta esperada for favorável e, de um a cinco, se ao contrário, a resposta esperada for desfavorável.

\section{Procedimentos}

Outros procedimentos relevantes para a construção e validação da escala são: (a) análise teórica prévia dos itens, realizada por dois tipos de juízes, técnicos (semântica) e experts (pertinência em relação ao construto). Pasquali (1999) e Pereira (1999) consideram suficiente a avaliação de cerca de seis juízes para realizar cada uma destas tarefas; (b) aplicação de aproximadamente dez pré-testes antes da aplicação efetiva do instrumento, em indivíduos com características similares às da população em estudo, para se detectar falhas na elaboração dos itens: ambiguidades; respostas não-previstas; dificuldade de compreensão pela ordem inversa ou negativa; e, (c) estudo piloto, que deve ser aplicado em uma amostra de aproximadamente 100 participantes para verificação da consistência da escala de atitudes (BARBETTA, 2002; BISQUERRA; SARRIERA; MARTINEZ, 2004; PASQUALI, 1999).

A técnica para a coleta dos dados consistiu na aplicação da escala em grupos, nas próprias salas de aula dos estudantes de cada curso. Os alunos receberam instruções padronizadas da pesquisadora e foram acompanhados pela mesma até o término da tarefa para possíveis esclarecimentos.

Antes da realização deste estudo, o projeto foi aprovado pelo Comitê de Ética para Pesquisa em Seres Humanos da Universidade Federal de Santa Catarina $\left(n^{\circ} 366 / 07\right)$, e todos os procedimentos obedeceram às diretrizes da Resolução 196/96 para pesquisa em seres humanos.

Para o tratamento estatístico dos dados obtidos a partir da escala, foram definidos os seguintes procedimentos de análise: (a) consistência interna entre os itens $(\alpha>0,75)$; (b) fatorial ou covariância para verificar a unidimensionalidade da escala (seleção de itens com cargas fatoriais acima de 0,50 ); (c) distribuição normal (z) que forneceu escores-padrão, indicando o quanto um dado sujeito se afasta da média e consequentemente, se ele tem uma atitude favorável ou desfavorável em relação ao atributo medido; e, (d) correlação entre escores de duas situações produzidas pelo mesmo teste, a partir dos dados do estudo-piloto comparados aos do estudo final - $\mathrm{r}$ aproximado a 0,90 (BARBETTA, 2002; BISQUERRA; SARRIERA; MARTINEZ, 2004; PASQUALI, 1999). Todos os dados foram analisados com auxílio do software SPSS (Statistic Package of Social Science, versão 10.0). 


\section{Resultados}

\section{Resultados da Construção e da Validação da EASE \\ Construção da EASE}

Os itens da escala para mensurar aspectos físicos, psicológicos, sociais e transtornos associados à saúde e à estética corporais foram elaborados a partir de ideias que estruturaram as representações sociais de estudantes de cursos superiores, conforme estudo realizado por Camargo et al. (2011), antes do início desta pesquisa - e também por alguns pensamentos contidos nas representações sociais que foram difundidas na mídia impressa a respeito do corpo, conforme outra pesquisa realizada por Goetz et al. (2008).

A partir desses dois estudos preliminares, os 60 itens da EASE foram selecionados e iniciou-se o processo de validação da escala (ALCHIERI; CRUZ, 2003). Os itens foram selecionados de forma a serem distribuídos proporcionalmente entre quatro fatores, conforme ilustra a Tabela 1.

Tabela 1 - Fatores, dimensões e descrição dos construtos do corpo

\begin{tabular}{|c|c|c|c|}
\hline Fatores & Dimensões & Descrição dos Construtos & Itens \\
\hline $\begin{array}{l}\text { Fator } 1 \text { - Aspectos } \\
\text { físicos da saúde e da } \\
\text { estética. }\end{array}$ & $\begin{array}{l}\text { - Cognitiva. } \\
\text { - Afetiva. } \\
\text { - Comportamental. }\end{array}$ & $\begin{array}{l}\text { Conhecimentos específicos, } \\
\text { posicionamento afetivo e } \\
\text { comportamentos em: } \\
\text { - nutrição, dieta, exercícios, beleza, } \\
\text { cirurgias, tratamentos, orientação } \\
\text { profissional. }\end{array}$ & $\begin{array}{l}15 \text { itens } \\
7,20,13,50 \\
25,10,22,42 \\
54,35,44,20 \\
5,30,17\end{array}$ \\
\hline $\begin{array}{l}\text { Fator } 2 \text { - Aspectos } \\
\text { psicológicos da saúde } \\
\text { e da estética. }\end{array}$ & $\begin{array}{l}\text { - Cognitiva. } \\
\text { - Afetiva. } \\
\text { - Comportamental. }\end{array}$ & $\begin{array}{l}\text { Conhecimentos específicos, } \\
\text { posicionamento afetivo e } \\
\text { comportamentos em: } \\
\text { - cirurgias, tratamentos, traços ou } \\
\text { características individuais, } \\
\text { satisfação, autoestima, orientação } \\
\text { profissional. }\end{array}$ & $\begin{array}{l}15 \text { itens } \\
48,57,6,12 \\
27,33,40,58 \\
45,31,47,11 \\
21,14,8\end{array}$ \\
\hline $\begin{array}{l}\text { Fator } 3 \text { - Aspectos } \\
\text { sociais da saúde e da } \\
\text { estética. }\end{array}$ & $\begin{array}{l}\text { - Cognitiva. } \\
\text { - Afetiva. } \\
\text { - Comportamental. }\end{array}$ & $\begin{array}{l}\text { Conhecimentos específicos, } \\
\text { posicionamento afetivo e } \\
\text { comportamentos em: } \\
\text { - interação e isolamento social, } \\
\text { suporte social (familiares, amigos, } \\
\text { colegas); inserção no mercado de } \\
\text { trabalho / capacidade / sucesso; } \\
\text { realização de atividades de lazer } \\
\text { (prazerosas). }\end{array}$ & $\begin{array}{l}15 \text { itens } \\
52,37,4,3,1 \\
9,16,19,24 \\
28,34,15,18 \\
23,26\end{array}$ \\
\hline $\begin{array}{l}\text { Fator } 4 \text { - Aspectos } \\
\text { biopsicossociais da } \\
\text { saúde e da estética }\end{array}$ & $\begin{array}{l}\text { - Cognitiva. } \\
\text { - Afetiva. } \\
\text { - Comportamental. }\end{array}$ & $\begin{array}{l}\text { Conhecimentos específicos, } \\
\text { posicionamento afetivo e } \\
\text { comportamentos em: } \\
\text { - transtornos dismórficos corporais; } \\
\text { transtornos obsessivo-compulsivos; } \\
\text { transtornos de ansiedade e } \\
\text { depressão; distúrbios alimentares; } \\
\text { tratamentos, cirurgias; orientação } \\
\text { profissional. }\end{array}$ & $\begin{array}{l}15 \text { itens } \\
32,36,59,38 \\
55,46,41,43 \\
60,51,39,49 \\
53,56,29\end{array}$ \\
\hline
\end{tabular}




\section{Validação}

Após a construção do modelo inicial da escala, iniciaram-se os processos de Validação de Face e de Construto. A análise teórica prévia dos itens foi realizada por dois tipos de juízes, seis deles técnicos, que avaliaram a semântica e outros seis experts, que analisaram a pertinência em relação ao construto (PASQUALI, 1999; PEREIRA, 1999). O modelo inicial da escala, com 60 itens, para mensurar atitudes de estudantes universitários em saúde e estética foi enviado aos 12 juízes para que fossem realizadas as análises, com esclarecimentos a respeito do público ao qual se destinava, constando também o enunciado que seria utilizado para orientar os estudantes e as opções de respostas (muito favorável; favorável; nem favorável, nem desfavorável; pouco favorável; desfavorável).

A partir das análises realizadas pelos juízes técnicos e experts, todas as sugestões de exclusão apontadas pelos mesmos foram consideradas, sendo que da escala original foram retirados 12 dos 60 itens iniciais, alguns por apresentarem demasiada semelhança com outros; pela falta de clareza; falta de simplicidade; falta de precisão nas sentenças ou modalidade (PASQUALI, 1999). Foram excluídos os itens de números 9, 10, 16, 19, 32, 38, 42, 45, 46, 49, 51, 52. Quanto à validade de construto, os juízes experts consideraram que todos os itens foram pertinentes e adequados a cada fator.

Após a exclusão dos itens mencionados, restaram 48 sentenças, para as quais os juízes sugeriram alterações na forma escrita para aprimoramento da clareza, da precisão e da simplicidade. Tiveram alterações os itens $3,4,5,7,8,11$, $12,14,15,16,20,21,22,23,24,25,26,27,30,31,33,35,37,38,40,42,43$, $44,45,46$ e 48 .

\section{Pré-Testes}

Foi realizada a coleta de dez pré-testes antes da aplicação efetiva da escala no Estudo Piloto, em indivíduos com características similares às da população em estudo, para se detectar falhas na elaboração dos itens. Após a aplicação dos pré-testes, o item 31 "Medicamentos sob orientação médica somente devem ser utilizados em casos graves de depressão, podendo trazer benefícios à saúde e à estética corporal" foi modificado por ter sido questionado por três dos 10 participantes desta etapa, que ficaram em dúvida quanto à resposta, e por ter sido considerada ambígua quanto à sua estrutura. $\mathrm{O}$ item foi substituído por "Antidepressivos utilizados sob prescrição médica em casos de depressão, podem trazer benefícios à saúde e à estética corporal”.

\section{Precisão}

O próximo passo realizado para validação da escala foi um estudo-piloto para a análise da consistência interna dos itens, pela aplicação do teste Alfa de Cronbach. Este teste permitiu a redução de itens na escala, visto que o alfa teve seu escore aumentado pela exclusão daqueles que apresentaram maior variabilidade entre as respostas. Para a aplicação do Teste Alfa de Cronbach, a escala de 48 itens foi aplicada em 100 participantes (BARBETTA, 2002; BISQUERRA; 
SARRIERA; MARTINEZ, 2004; PASQUALI, 1999), sendo 25 deles de cada curso que compôs a amostra. Após a aplicação deste teste de Cronbach, restaram 28 itens na escala, sendo aqueles que apresentaram maior estabilidade ou consistência entre os resultados. Obteve-se um valor de 0,7648 do alfa. Para análise da consistência, valores iguais ou superiores a 0,75 são considerados suficientemente altos (BARBETTA, 2002). Esta versão da EASE, com 28 itens, foi utilizada para a coleta de dados com os 400 participantes do estudo final.

\section{Análise Fatorial}

A primeira análise fatorial foi realizada sem a definição prévia de fatores. A análise de fatores foi realizada pelo programa estatístico SPSS, 10.0, que definiu quatro fatores para serem retidos. Na Figura 1, apresenta-se o diagrama de declividade, cujos resultados são indicativos de que o início da rampa suave não está bem definido, confirmando o que já foi visto na análise fatorial inicial, com relação à distribuição dos itens primeiramente em quatro fatores.

\section{Diagrama de Declividade}

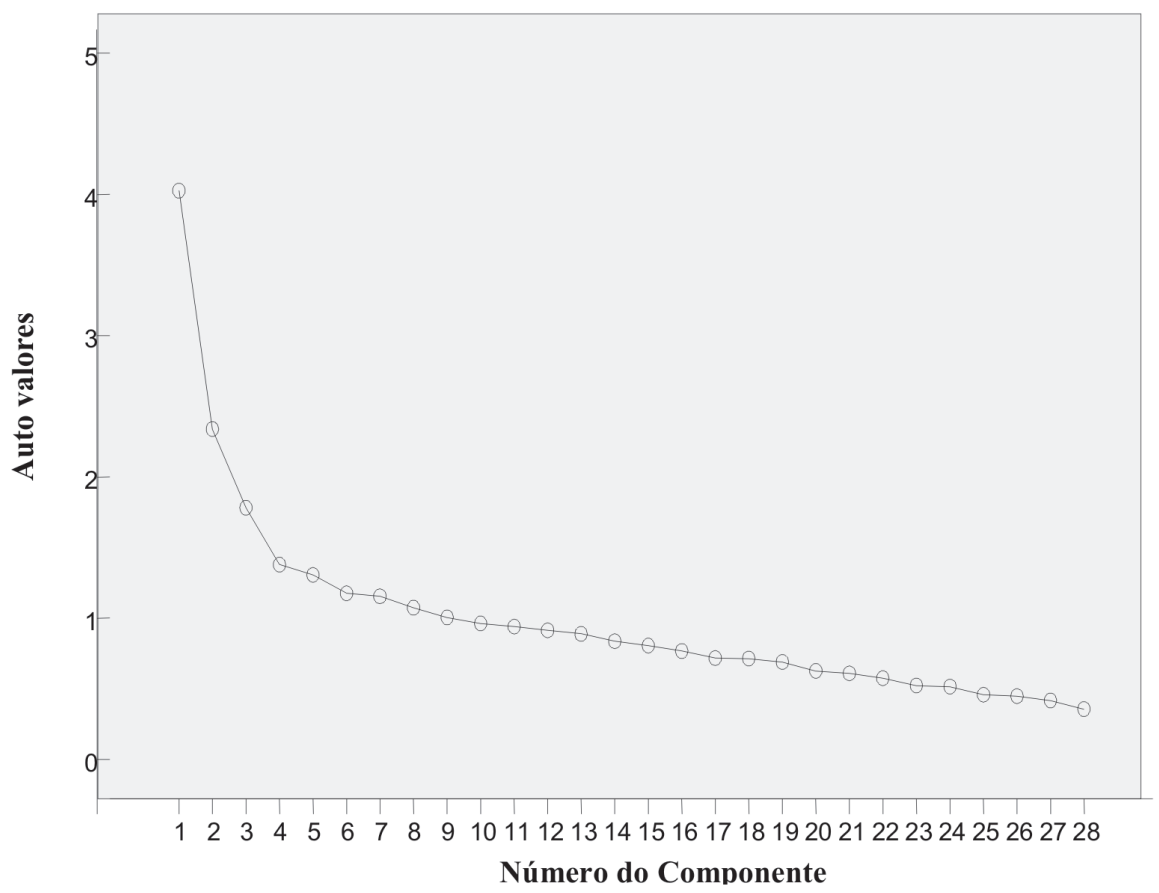

Figura 1. Diagrama de declividade da análise fatorial inicial

Os autovalores mostram a proporção da variância de aproximadamente $40 \%$, que cada fator é capaz de manter, nesse caso. Contudo, no Fator 3, referente aos Aspectos Sociais, somente uma variável teve carga fatorial satisfatória (acima de 0,40). Não cabe explicar mais uma variância quando somente 
uma variável tem carga considerável em um fator (DANCEY; REIDY, 2006). Justifica-se, portanto, que somente três fatores sejam considerados para a análise fatorial, sendo estes os fatores 1 (Aspectos Físicos), 2 (Aspectos Psicológicos) e 4 (Aspectos Biopsicossociais).

Os resultados desses dois procedimentos estatísticos realizados anteriormente justificam a opção pelo método de rotação Varimax, cujo objetivo é maximizar as altas correlações e minimizar as baixas (DANCEY; REIDY, 2006). Na Tabela 2, apresentam-se os resultados da análise fatorial realizada pelo método Varimax, na qual se definiu previamente a verificação da escala com três fatores.

Tabela 2 - Resultados da análise fatorial Varimax

\begin{tabular}{|c|c|c|c|c|}
\hline $\begin{array}{l}\mathrm{N}^{\circ} \mathrm{do} \\
\text { Item }\end{array}$ & Item & $\begin{array}{c}\text { Fator } 1 \\
\mathbf{R}\end{array}$ & $\begin{array}{c}\text { Fator } 2 \\
\quad \mathbf{r}\end{array}$ & $\begin{array}{c}\text { Fator } 3 \\
\mathbf{r}\end{array}$ \\
\hline 14 & A prática regular de exercícios ajuda a combater a depressão. & 0,709 & & \\
\hline 4 & $\begin{array}{l}\text { Praticar exercícios regularmente também é uma forma de } \\
\text { lazer. }\end{array}$ & 0,657 & & \\
\hline 32 & Praticar esportes coletivos ajuda a fazer amigos. & 0,582 & & \\
\hline 35 & $\begin{array}{l}\text { Uma hora e meia de exercícios físicos orientados por um } \\
\text { profissional (realizados } 2 \text { ou } 3 \text { vezes por semana), são uma } \\
\text { prática saudável para manter a forma. }\end{array}$ & 0,557 & & \\
\hline 45 & O humor afeta a saúde mental e a estética corporal. & 0,479 & & \\
\hline 47 & $\begin{array}{l}\text { Tratamento psicológico pode ajudar a pessoa a melhorar a } \\
\text { autoestima. }\end{array}$ & 0,436 & & \\
\hline 12 & $\begin{array}{l}\text { Tratamentos clínicos para melhorar a forma corporal devem } \\
\text { ser indicados antes dos cirúrgicos. }\end{array}$ & 0,416 & & \\
\hline 3 & $\begin{array}{l}\text { Fazer e manter novas amizades ajudam no bem-estar da } \\
\text { pessoa. }\end{array}$ & 0,401 & & \\
\hline 33 & Tratamentos estéticos são mera futilidade. & & 0,698 & \\
\hline 27 & Tratamentos estéticos podem aiudar a melhorar a autoestima. & & 0,655 & \\
\hline 44 & $\begin{array}{l}\text { Cuidados com a beleza corporal podem trazer benefícios à } \\
\text { saúde de uma pessoa. }\end{array}$ & 0,622 & & \\
\hline 48 & $\begin{array}{l}\text { Cirurgia estética é uma opção adequada quando a pessoa tem } \\
\text { problemas com a imagem corporal. }\end{array}$ & & 0,619 & \\
\hline 9 & $\begin{array}{l}\text { Pequenos cuidados de beleza (pintar unhas, cortar cabelos, } \\
\text { usar perfume) podem melhorar o humor. }\end{array}$ & & 0,607 & \\
\hline 2 & $\begin{array}{l}\text { Cirurgias estéticas quando necessárias podem trazer } \\
\text { benefícios à saúde. }\end{array}$ & & 0,561 & \\
\hline 16 & $\begin{array}{l}\text { Mesmo sem orientação médica, usar laxante é um hábito } \\
\text { adequado para perder peso. }\end{array}$ & & & 0,553 \\
\hline 20 & $\begin{array}{l}\text { Restringir alimentos sem orientação profissional é uma forma } \\
\text { saudável para perder peso. }\end{array}$ & & & 0,542 \\
\hline 25 & Todas as pessoas magras são saudáveis. & & & 0,462 \\
\hline 26 & $\begin{array}{l}\text { Medicamentos para aumentar a massa muscular (esteróides e } \\
\text { anabolizantes) são uma opção saudável para alcançar o corpo } \\
\text { desejado. }\end{array}$ & & & 0,458 \\
\hline 15 & Somente a beleza não sustenta amizades duradouras. & & & 0,430 \\
\hline 18 & $\begin{array}{l}\text { A beleza de uma pessoa não se relaciona com a capacidade } \\
\text { dela para o trabalho. }\end{array}$ & & & 0,425 \\
\hline
\end{tabular}

$\mathrm{N}=400$

Fractal, Rev. Psicol., v. 26 - n. 1, p. 199-222, Jan./Abr. 2014 
Dos 28 itens utilizados inicialmente para a análise fatorial, restaram 20, distribuídos em três fatores. Pasquali (1999) considera ideal um número aproximado a 20 itens para o instrumento escalar final. Utilizou-se como critério para excluir os itens sua carga fatorial nos fatores, de modo que foram mantidos somente aqueles com valores superiores a $0,40(\mathrm{r} \geq 0,4)$.

Após a análise fatorial, observou-se que dentre os itens que apresentam maior carga fatorial estão respectivamente: "A prática regular de exercícios ajuda a combater a depressão", "Praticar exercícios regularmente também é uma forma de lazer", "Cuidados com a beleza corporal podem trazer benefícios à saúde de uma pessoa", "Tratamentos estéticos são mera futilidade", "Tratamentos estéticos podem ajudar a melhorar a autoestima", "Cirurgia estética é uma opção adequada quando a pessoa tem problemas com a imagem corporal" e "Pequenos cuidados de beleza (pintar unhas, cortar cabelos, usar perfume) podem melhorar o humor". Os três primeiros itens de carga fatorial mais forte são pertencentes ao Fator 1 (Aspectos de Saúde) e, os outros quatro, são oriundos do Fator 2 (Aspectos Psicológicos). Segundo Dancey e Reidy (2006), os demais itens podem ser considerados válidos porque apresentam cargas fatoriais consideradas suficientes, que devem ser de no mínimo $0,3(\mathrm{r} \geq \pm 0,3)$.

\section{Fidedignidade}

Para verificar a fidedignidade dos resultados, foi utilizado o procedimento estatístico de distribuição normal (z), para identificar qual a média de respostas no grupo. As respostas esperadas na EASE devem assumir valores entre 4 e 5, ou seja, indicativos de atitudes favoráveis; ou desfavoráveis, quando da ordem inversa das questões. Nesse sentido, as três medidas de tendência central se aproximaram de uma curva normal, com valores bastante próximos entre média, moda e mediana $(\mathrm{M}=4,42 ; \mathrm{DP}=0,29 ; \mathrm{Md}=4,45 ; \mathrm{Mo}=4,50)$. Na Figura 2, são ilustrados os resultados da distribuição normal. 


\section{Distribuicão Normal}

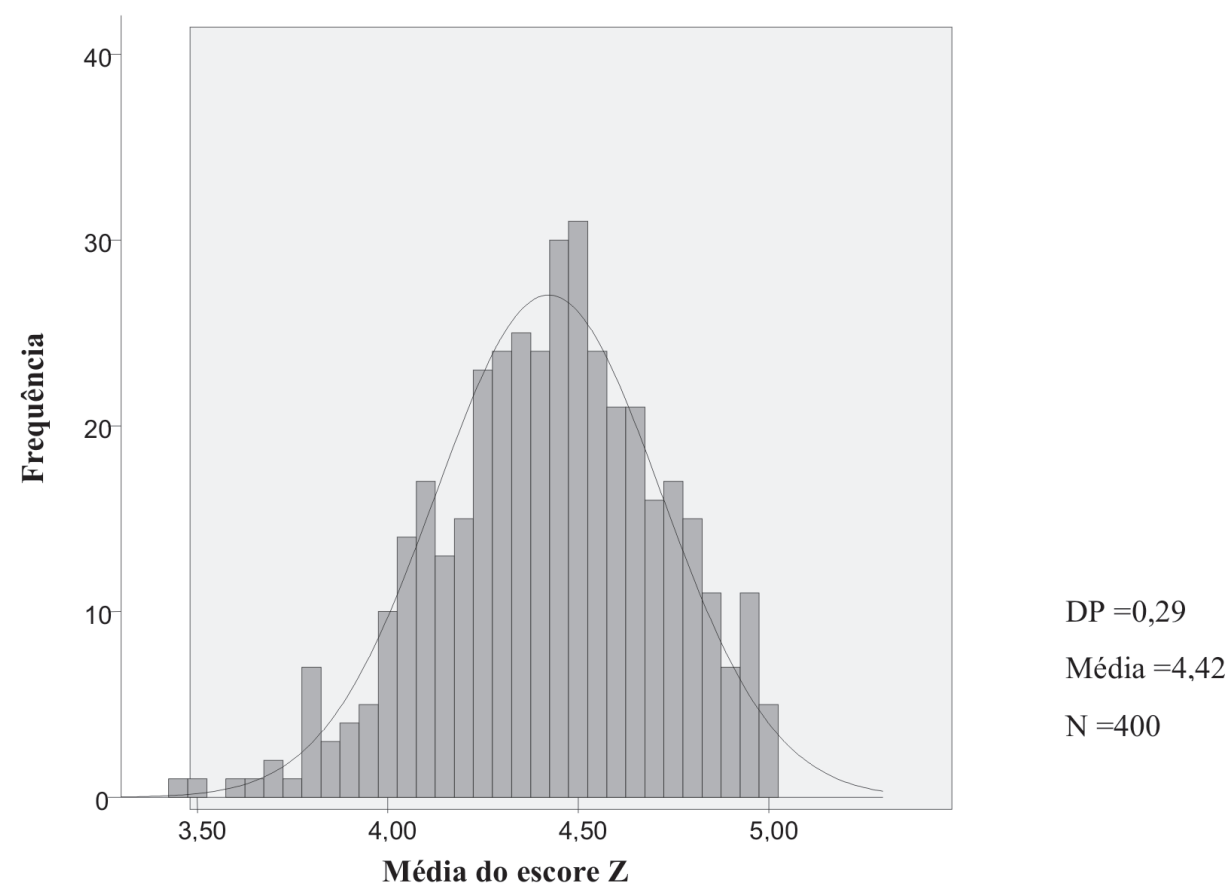

Figura 2 - Histograma da distribuição normal das médias na EASE

Pela Figura 2 pode-se visualizar a distribuição das respostas dos sujeitos. A média obtida na distribuição normal foi de 4,42 e o desvio-padrão de 0,29 , indicativos de que nesta amostra apenas os sujeitos com escores inferiores a 4,12 estariam fora da curva de normalidade, isto é, em torno de $15 \%$.

Na Tabela 3 são apresentadas as médias e os respectivos desvios-padrão, obtidos a partir da análise das respostas de todos os participantes na escala EASE, para verificar as atitudes dos universitários perante as difusões midiáticas a respeito do corpo. 
Tabela 3 - Descrição de resultados obtidos pela EASE

\begin{tabular}{|c|c|c|c|}
\hline $\begin{array}{l}N^{0} \text { do } \\
\text { Item }\end{array}$ & Item & M & DP \\
\hline 2 & $\begin{array}{l}\text { Cirurgias estéticas quando necessárias podem trazer benefícios à } \\
\text { saúde. }\end{array}$ & 4,05 & 0,89 \\
\hline 3 & Fazer e manter novas amizades ajudam no bem-estar da pessoa. & 4,76 & 0,51 \\
\hline 4 & Praticar exercícios regularmente também é uma forma de lazer. & 4,66 & 0,63 \\
\hline 9 & $\begin{array}{l}\text { Pequenos cuidados de beleza (pintar unhas, cortar cabelos, usar } \\
\text { perfume) podem melhorar o humor. }\end{array}$ & 4,38 & 0,80 \\
\hline 12 & $\begin{array}{l}\text { Tratamentos clínicos para melhorar a forma corporal devem ser } \\
\text { indicados antes dos cirúrgicos. }\end{array}$ & 4,40 & 0,79 \\
\hline 14 & A prática regular de exercícios ajuda a combater a depressão. & 4,61 & 0,63 \\
\hline 15 & Somente a beleza não sustenta amizades duradouras. & 4,58 & 0,91 \\
\hline 16 & $\begin{array}{l}\text { Mesmo sem orientação médica, usar laxante é um hábito adequado } \\
\text { para perder peso. }\end{array}$ & 1,15 & 0,57 \\
\hline 18 & $\begin{array}{l}\text { A beleza de uma pessoa não se relaciona com a capacidade dela para } \\
\text { o trabalho. }\end{array}$ & 4,16 & 1,17 \\
\hline 20 & $\begin{array}{l}\text { Restringir alimentos sem orientação profissional é uma forma } \\
\text { saudável para perder peso. }\end{array}$ & 1,48 & 0,75 \\
\hline 25 & Todas as pessoas magras são saudáveis. & 1,32 & 0,65 \\
\hline 26 & $\begin{array}{l}\text { Medicamentos para aumentar a massa muscular (esteróides e } \\
\text { anabolizantes) são uma opção saudável para alcançar o corpo } \\
\text { desejado. }\end{array}$ & 1,14 & 0,50 \\
\hline 27 & Tratamentos estéticos podem ajudar a melhorar a autoestima. & 4,19 & 0,79 \\
\hline 32 & Praticar esportes coletivos ajuda a fazer amigos. & 4,50 & 0,71 \\
\hline 33 & Tratamentos estéticos são mera futilidade. & 1,88 & 0,91 \\
\hline 35 & $\begin{array}{l}\text { Uma hora e meia de exercícios físicos orientados por um profissional } \\
\text { (realizados } 2 \text { ou } 3 \text { vezes por semana), são uma prática saudável para } \\
\text { manter a forma. }\end{array}$ & 4,53 & 0,67 \\
\hline 44 & $\begin{array}{l}\text { Cuidados com a beleza corporal podem trazer benefícios à saúde de } \\
\text { uma pessoa. }\end{array}$ & 4,20 & 0,75 \\
\hline 45 & O humor afeta a saúde mental e a estética corporal. & 4,45 & 0,66 \\
\hline 47 & Tratamento psicológico pode ajudar a pessoa a melhorar a autoestima. & 4,52 & 0,66 \\
\hline 48 & $\begin{array}{l}\text { Cirurgia estética é uma opção adequada quando a pessoa tem } \\
\text { problemas com a imagem corporal. }\end{array}$ & 3,47 & 1,10 \\
\hline
\end{tabular}

$\mathrm{N}=400$

Pela análise das médias dos itens da EASE, pode-se concluir que esses resultados são indicativos de que em sua maioria, os estudantes têm atitudes que variam entre "muito favorável" e "favorável" em relação a práticas e hábitos considerados como adequados em relação aos cuidados com beleza e saúde, incluindo nestes, os aspectos psicológicos e sociais. Em contrapartida, apresentam atitudes que variam entre "pouco favorável" e "desfavorável", apenas nos itens $16,20,25$ e 26 , que dizem respeito a práticas inadequadas ou radicais relacionadas à saúde e à estética do corpo, ou ainda direcionada a mensurar juízo de valores quanto à estética corporal (item 33).

Além disso, a escala que havia sido originalmente construída com quatro fatores, que abrangiam respectivamente: (Fator 1) aspectos físicos; (Fator 2) psicológicos; (Fator 3) sociais; e, (Fator 4) biopsicossociais; pela análise fatorial, teve o Fator três excluído, sendo que o Fator 4, aspectos biopsicosso- 
ciais foi renomeado como Fator 3. Na Tabela 4, são apresentados os valores da média geral e desvio-padrão da EASE, assim como esses mesmos valores obtidos pela análise de cada fator.

Tabela 4 - Escores de medidas da EASE

\begin{tabular}{lcccc}
\multicolumn{1}{c}{ Escores da EASE } & M & DP & Mo & Md \\
\hline Escala geral & 3,62 & 0,25 & 3,70 & 3,65 \\
Fator 1 - Aspectos físicos da saúde e da estética & 4,51 & 0,37 & 5,00 & 4,55 \\
$\begin{array}{l}\text { Fator 2 - Aspectos psicológicos da saúde e da } \\
\text { estética }\end{array}$ & 3,50 & 0,44 & 3,60 & 3,60 \\
$\begin{array}{l}\text { Fator 3 - Aspectos biopsicossociais da saúde e da } \\
\text { estética }\end{array}$ & 2,30 & 0,32 & 2,33 & 2,33 \\
\hline
\end{tabular}

$\mathrm{N}=400$

A média obtida a partir de todos os itens da EASE foi de 3,62 (DP = 0,25), indicativa de que os participantes deste estudo apresentam posicionamento intermediário entre ser "nem favorável, nem desfavorável" e "favorável" em relação à saúde e estética do corpo, o que demonstra que os estudantes têm atitude levemente positiva frente a esses aspectos investigados. $\mathrm{O}$ valor mais intermediário $(\mathrm{Md})$ aproximou-se da média e o valor que mais apareceu (Mo) ficou um pouco acima da média. Pelas medidas de tendência central e de dispersão da escala, observa-se atitude levemente positiva dos participantes em relação à saúde e à estética corporal.

Em relação ao Fator 1, que agrupou aspectos físicos relativos à saúde e à estética corporal, o posicionamento dos estudantes é intermediário entre ser "favorável" e "muito favorável", observado pela média 4,52 ( $\mathrm{DP}=0,37)$, indicativo de atitudes positivas. $\mathrm{O}$ valor que apareceu com mais frequência foi de estudantes com atitude muito favorável; e, o valor intermediário aproxima-se da média. Tais resultados corroboram uma hipótese de atitudes adequadas dos estudantes em relação a cuidados físicos e práticas inerentes à saúde do corpo e à estética corporal.

No que se refere ao Fator 2, sobre aspectos psicológicos implicados em saúde e estética do corpo, obteve-se média 3,50 $(\mathrm{DP}=0,44)$, indicativa de que os participantes demonstram atitudes que variam entre "nem favorável, nem desfavorável" e "favorável", indicando uma atitude que varia entre neutra e levemente positiva. Esperava-se nesse fator, atitudes positivas dos estudantes relativas a aspectos psicológicos implicados nos cuidados com saúde e estética do corpo.

Quanto ao Fator 3, que investigou aspectos biopsicossociais relacionados à saúde e à estética corporal, a média obtida foi de $2,30(\mathrm{DP}=0,32)$, cujo posicionamento dos participantes indica uma atitude levemente negativa, variando entre "nem favorável, nem desfavorável" e "pouco favorável". Entretanto, tais resultados corroboram a hipótese de um posicionamento condizente com práticas e hábitos adequados em saúde e estética por parte dos estudantes, visto que, a maioria dos itens deste fator foi construída de forma que a expectativa de resposta resultasse em posicionamento negativo. 
Realizou-se uma estimativa de pontuação da escala para a utilização prática da mesma, que pode servir como instrumento para avaliação complementar em casos de verificação de possíveis transtornos relacionados à saúde e estética corporais. Pela estimativa, espera-se que os indivíduos marquem as opções "muito favorável" e "favorável", que correspondem respectivamente aos números cinco e quatro, para cada item em que se espera atitude positiva (itens: 1, 2, 3, 4, 5, $6,7,9,13,14,16,17,18,19$ e 20); visto que nestes, são contempladas práticas, hábitos e cuidados considerados adequados e também envolvem aspectos sociais e psicológicos esperados. Para os demais itens, os indivíduos devem marcar somente a opção "desfavorável", pois se tratam de atitudes que envolvem hábitos e práticas corporais inadequadas ou consideradas como de risco para a saúde e, portanto, devem ser negativas (itens: $8,10,11,12$ e 15). Pela soma de todas as respostas esperadas por item, se os escores finais forem obtidos entre 60 e 80 pontos, considera-se que o indivíduo apresenta características salutares, no sentido de menor risco para práticas e hábitos desfavoráveis à saúde; enquanto que, escores entre 81 e 100 pontos podem estar associados a maior risco.

Para confirmar o posicionamento deste grupo frente a atitudes salutares em saúde e estética corporal, foi realizado o somatório de respostas de todos os participantes nos itens da EASE, e depois dividido pelo número de itens da escala, a partir do que se obteve a média $72,4\left(\sum=1448,25 ; \mathrm{DP}=4,90\right)$. Esperava-se que a média do grupo ficasse entre 60 e 80 pontos pela estimativa, considerando-se respostas adequadas ao nível ótimo de cuidados, práticas, valores e hábitos envolvendo saúde e estética e, consequentemente sem riscos para a saúde. Então, a média obtida pelo somatório de todos os itens traz implícito um valor dentro do que era esperado, considerando-se cuidados, conceitos, valores e práticas de beleza e saúde corporal destes estudantes.

Para verificar relações entre os resultados da EASE e os dados sociodemográficos coletados no estudo, foram utilizadas as seguintes análises: Para comparar a variável sexo com a média da escala EASE, utilizou-se o teste t. Foi encontrada diferença estatisticamente significativa entre homens $(M=3,66)$ e mulheres $(\mu=3,60)$ em relação ao posicionamento na escala EASE, $t(399)=$ 75,$89 ; p=0,001$; Então a variável sexo determina diferenças entre atitudes em relação à saúde e a estética corporal destes participantes.

Para comparar a idade (categorizada por faixas etárias, com intervalos de cinco anos a contar da idade mínima até a máxima) dos participantes com o posicionamento na EASE (obtido pela média geral), utilizou-se o teste de ANOVA, não houve diferença estatisticamente significativa entre os estudantes quanto ao posicionamento na escala quando comparado por faixas etárias, $F(3,394)=$ 0,$098 ; p=0,961$.A ANOVA também foi utilizada para comparar a média geral obtida na EASE em função da variável curso dos estudantes, e não revelou diferença estatística entre o posicionamento dos estudantes na escala relacionado ao curso dos mesmos, $F(3,396)=1,34 ; p=0,261$. 


\section{Conclusões}

Todos os processos e análises estatísticas realizadas para a construção e validação da EASE, assim como os resultados obtidos a partir de sua aplicação com os estudantes universitários participantes deste estudo, sugerem que a referida escala constitui um instrumento válido e confiável para a mensuração de atitudes em saúde e em estética corporal, podendo ser reproduzida com resultados semelhantes aos encontrados nesta pesquisa, desde que a população-alvo tenha características semelhantes às desta (PASQUALI, 1999; DANCEY; REIDY, 2006).

Os participantes posicionam-se de maneira levemente favorável na escala EASE, o que indica que em relação a aspectos que envolvem saúde e estética corporal, apresentam atitudes predominantemente favoráveis a cuidados, práticas, valores e hábitos relativos ao corpo. Apesar da influência da mídia como potencial vetor de difusão de modelos corporais ideais, esperava-se que estes participantes apresentassem cuidados adequados com a estética e a saúde do corpo, considerando-se o nível de esclarecimentos inclusive relativos à saúde da maioria destes estudantes pelos próprios cursos que foram investigados e pelo nível de escolaridade.

No que tange aos fatores da EASE, observou-se: quanto ao Fator 1, que agrupou predominantemente aspectos físicos e práticos relativos à saúde e à estética corporal, os estudantes apresentaram posicionamento aproximado ao "muito favorável", indicando que nas questões físicas e práticas relativas ao corpo, têm atitudes muito positivas; no Fator 2, sobre aspectos psicológicos implicados em saúde e estética do corpo, novamente a atitude dos participantes está aproximada da positiva; e pelo Fator 3, que investigou aspectos biopsicossociais relacionados à saúde e à estética corporal, o posicionamento dos participantes indica uma atitude levemente negativa, corroborando o posicionamento condizente com práticas e hábitos adequados em saúde e estética por parte dos estudantes, visto que, a maioria dos itens deste fator foi construída de forma que a expectativa de resposta resultasse em posicionamento negativo. Nesse sentido, observa-se a dimensão avaliativa das atitudes em relação ao objeto corpo; segundo Rodrigues, Assmar e Jablonski (2002), estas são predisposições aprendidas e relativamente duradouras para responder favorável ou desfavoravelmente a um fenômeno.

O conceito de saúde envolve o bem-estar em diversos e complexos aspectos da vida dos indivíduos, que no mínimo perpassam pelo biológico, psicológico e social. Neste estudo, pretendeu-se contemplar esses aspectos mínimos, sem desconsiderar a complexidade e a amplitude de cada aspecto envolvido na saúde, afirma-se que os participantes apresentam um nível adequado de cuidados, práticas, valores e hábitos envolvendo saúde e estética corporal. Isso pelos resultados obtidos e comparados com a estimativa de pontuação esperada na EASE. Contudo, Czeresnia e Freitas (2004) consideram difícil significar integralmente o conceito de saúde, tendo em vista que há fatores singulares e subjetivos que a determinam.

Homens e mulheres neste estudo se posicionam de maneira diferente em questões de saúde e estética corporal, quando avaliados pela escala EASE. Nesse sentido, o fato de as mulheres se sentirem menos satisfeitas do que os homens 
em relação ao corpo, pode ter sido determinante para a diferença entre as atitudes encontradas entre ambos sobre saúde e estética. Corroborando os resultados deste estudo, Castilho (2001) considera que as mulheres são mais insatisfeitas do que os homens com sua imagem corporal, em especial as mais jovens, o que pode estar relacionando ao fato de que a mídia projeta mais enfaticamente a atração física do corpo da mulher e como valor associado ao sexo feminino.

Então, de um modo geral, os estudantes tendem a declarar atitudes positivas em saúde e estética corporal, ainda que em algumas questões sejam evidentes diferenças determinadas pelo sexo, sendo as mulheres mais propensas à adesão a tratamentos mais radicais (como no caso da cirurgia plástica estética) que os homens, expondo-se, portanto, a maior risco (GOETZ, 2009).

Especula-se que a aplicabilidade desta escala se estenda a públicos com igual nível de escolaridade aos dos participantes deste estudo e também àqueles com menor grau de instrução, desde que os itens possam ser adequadamente compreendidos pelo avaliando, e nisso, o psicólogo avaliador pode auxiliar, fornecendo esclarecimentos referentes ao mesmo.

Nesse sentido, considerando-se que a EASE consiste em instrumento que se comprovou por este estudo ser válido e confiável para a mensuração de atitudes em saúde e em estética corporal, especificamente no que diz respeito aos três fatores pertinentes a este construto - físicos, psicológicos e biopsicossociais, pode ser utilizada como recurso complementar e esclarecedor em casos de avaliação de pacientes com TDCs, obesidade mórbida e demais casos de insatisfação com o corpo ou a imagem corporal.

A construção e a validação da EASE, justificam-se pelo aumento dos TDCs, casos de obesidade e demais questões de saúde que se relacionam à estética corporal, na atualidade, possibilitando a produção de dados mais assertivos para a adequada avaliação dos pacientes que necessitam de tratamentos radicais e invasivos, como no caso da utilização de medicamentos controlados e de intervenções cirúrgicas. A avaliação psicológica tem sido uma prática constante em casos de psicopatologias que envolvem o binômio "saúde-estética", assim como uma exigência do SUS, para a realização de cirurgias bariátricas, cuja finalidade principal é a de garantir o bem-estar biopsicossocial dos sujeitos no período pós-cirúrgico.

Outro aspecto de relevância consiste no fato de que dentre os testes e instrumentos de avaliação psicológica regulamentados pelo Conselho Federal de Psicologia, não há nenhum especificamente voltado à análise de diversos problemas ou transtornos que relacionam saúde e estética. 


\section{REFERÊNCIAS}

ABREU, C. N.; CANGELLI FILHO, R. Anorexia nervosa e bulimia nervosa: abordagem cognitivo-construtivista de psicoterapia. Revista de Psiquiatria Clínica, [S.1.], v. 31, n. 4, p. 177-183, 2004.

AJZEN, I. Nature and operation of attitudes. Annual Reviews of Psychology, [S.1.], v. 52, p. 27-58, 2001.

ALCHIERI, J. C.; CRUZ, R. M. Avaliação psicológica: conceito, métodos e instrumentos. ed. rev. São Paulo: Casa do Psicólogo, 2003.

ANDRIEU, B. Le dictionnaire du corps em sciences humaines e sociales. Paris: CNRS, 2006.

ASSUNÇÃO, S. S. M. Dismorfia muscular. Revista Brasileira de Psiquiatria, [S.1.], v. 24, n. 3, p. 80-84, 2002.

BARBETTA, P. A. Estatística aplicada às ciências sociais. ed. rev. Florianópolis: UFSC, 2002.

BISQUERRA, R.; SARRIERA, J. C.; MARTINEZ, F. Introdução à estatística: enfoque informático com o pacote estatístico SPSS. Porto Alegre: Artmed, 2004.

BRASIL. Ministério da Saúde. Políticas Públicas (PPMS: Portaria 628/2001). 2001. Disponível em: <http://dtr2001.saude.gov.br/sas/PORTARIAS/ Port2001/GM/GM-628.htm>. Acesso em: 20 out. 2007.

BRAUNER, V. L. P.; GOMES, S. B.; LOPES, D. M. Escultores de corpos: a representação do belo para os profissionais de saúde e beleza. In: CONGRESSO BRASILEIRO DE CIÊNCIAS DO ESPORTE, 15., 2007, Recife; CONGRESSO INTERNACIONAL DO ESPORTE, 2., 2007, Recife. Trabalho completo... Recife: CONBRACE, 2007.

BUENO, M. B.; FISBERG, R. M. Comparação de três critérios de classificação de sobrepeso e obesidade entre pré-escolares. Revista Brasileira de Saúde MaternoInfantil, [S.1.], v. 6, n. 4, p. 411-418, 2006.

CALAF, M. et al. Inventario de imagen corporal para feminas adolescentes (IICFA). Revista Interamericana de Psicologia / Internacional Journal of Psychology, v. 39, n. 3, p. 347-354, set./dez. 2005.

CAMARGO, B. V. A televisão como vetor de difusão de informações sobre a AIDS. In: COUTINHO, M. L. P. et al. (Org.). Representações sociais: abordagem interdisciplinar. João Pessoa: UFPB, 2003. p. 130-152. 
CAMARGO, B. V.; GOETZ, E. R.; BARBARÁ, A. S. Representação social da beleza de estudantes de Moda. In: JORNADA INTERNACIONAL, 4., 2005, João Pessoa; CONFERÊNCIA BRASILEIRA SOBRE REPRESENTAÇÕES SOCIAIS, 2., 2005, João Pessoa. Anais... João Pessoa: Universidade Federal da Paraíba, 2005a. p. 607-608.

CAMARGO, B. V.; GOETZ, E. R.; BARBARÁ, A. S. Representação social da beleza de estudantes de Moda. In: JORNADA INTERNACIONAL, 4., 2005, João Pessoa; CONFERÊNCIA BRASILEIRA SOBRE REPRESENTAÇÕES SOCIAIS, 2., 2005, João Pessoa. Anais... João Pessoa: Universidade Federal da Paraíba, 2005b, p. 3353-3362.

CAMARGO, B. V.; GOETZ, E. R.; JUSTO, A. M. Representação social da beleza de estudantes de Educação Física e de Moda. In: JORNADA INTERNACIONAL, 5., 2007, Brasília; CONFERÊNCIABRASILEIRASOBRE REPRESENTAÇÕES SOCIAIS, 3., 2007, Brasília. Anais... Brasília: [s.n.], 2007. p. 607-608.

CAMARGO, B. V. et al. Representações sociais do corpo em estética e saúde. Temas em Psicologia, [S.1.], v. 19, n. 1, p. 257-268, 2011.

CAMBRAIA, R. P. B. Aspectos psicobiológicos do comportamento alimentar. Revista de Nutrição, [S.1.], v. 17, n. 2, p. 217-225, abr./jun. 2004.

CASTILHO, S. M. A Imagem Corporal. Santo André: ESETec, 2001.

CLARKE, L. H.; GRIFFIN, M. The body natural and the body unnatural: beauty work and aging. Journal of Aging Studies, [S.1.], v. 27, n. 3, p. 197-210, 2009.

CONTARELLO, A.; FORTUNATI, L. ICTs and the human body: a social representation approach. In: LAW, P.-I.; FORTUNATI, L.; YANG, S. (Org.). New technologies in global societies. New Jersey: World Scientific, 2006. p. 51-74.

CZERESNIA, D.; FREITAS, C. M. (Org.). Promoção da saúde: conceitos, reflexões, tendências. Rio de Janeiro: Fiocruz, 2004.

DANCEY, C. P.; REIDY, J. Estatística sem matemática para psicologia. Tradução de Lorí Vialli. Porto Alegre: Artmed, 2006.

ESPÍNDOLA, C. R.; BLAY, S. L. Bulimia e transtorno da compulsão alimentar periódica: revisão sistemática e matassíntese. Revista de Psiquiatria do Rio Grande do Sul, [S.1.], v. 28, n. 3, p. 265-275, 2006.

ESPÍNDOLA, C. R.; BLAY, S. L. Percepção de familiares sobre a anorexia e a bulimia: revisão sistemática. Revista Saúde Pública, [S.1.], v. 43, n. 4, p. 707-716, 2009. 
GOETZ, E. R. Beleza e plasticomania. Curitiba: Juruá, 2013.

GOETZ, E. R. Representações sociais do corpo, mídia e atitudes. Tese (Doutorado em Psicologia)-Programa de Pós-Graduação em Psicologia, Universidade Federal de Santa Catarina, Florianópolis, 2009.

GOETZ, E. R. et al. Representação social do corpo na mídia impressa. Revista Psicologia e Sociedade, [S.1.], v. 20, n. 2, p. 226-236, 2008.

GOLDENBERG, M. Gênero e corpo na cultura brasileira. Psicologia Clínica, [S.1.], v. 17, n. 2, p. 65-80, 2005.

HUBERT, A.; DE LABARRE, M. La dictature de la minceur. Cahier de Nutrition et Diététique, [S.1.], v. 40, n. 6, p. 300-306, dec. 2005.

JODELET, D. La représentation du corps, ses enjeux privés et sociaux. In: HAINARD, J.; KAEHR, R. (Org.). Le corps enjeu. Neuchâtel: Secrétariat de L'Université, 1983. p. 127-140.

JODELET, D. La representación social: fenómenos, concepto y teoria. In: MOSCOVICI, S. (Org.). Psicologia Social: pensamiento y vida social. Barcelona: Paidós, 1986. v. 2, p. 469-494.

JODELET, D. Le corps, la persone et autrui. In: MOSCOVICI, S. (Org.). Psychologie sociale dês relations à autrui. Paris: Nathan, 1994. p. 41-68.

JODELET, D. (Org.). Representações sociais: um domínio em expansão In: . As representações sociais. Tradução de Lilian Ulup. Rio de Janeiro: Eduerj, 2001. p. 187-203.

JODELET, D. et al. Systeme de representation du corps et groupes sociaux. Paris: Laboratoire de Psychologie Sociale - E. H. E. S. S, 1982. Relatório v. 1.

KNAPP, M. L.; HALL, J.A. Os efeitos da aparência física e do odor na comunicação humana. In: JSN, 1999. p. 101-141. Comunicação não-verbal na interação humana. São Paulo:

LATTERZA, A. R. et al. Tratamento nutricional dos transtornos alimentares. Revista de Psiquiatria Clínica, [S.1.], v. 31, n. 4, p. 173-176, 2004.

LORDELO, R. A. et al. Eixos hormonais na obesidade: causa ou efeito? Arquivo Brasileiro de Endocrinologia e Metabolismo, [S.1.], v. 51, n. 1, p. 34-41, fev. 2007. 
LOTUFO, P. A. Determinantes novos da epidemia de obesidade no Brasil. Diagnóstico e Tratamento, [S.1.], v. 11, n. 3, p. 175-176, 2006.

MALYSSE, S. Um ensaio de antropologia visual do corpo ou como pensar em imagens o corpo visto? In: LYRA, B.; GARCIA, W. (Org.). Corpo e imagem. São Paulo: Arte \& Ciência, 2002. p. 132-141.

MANER, J. K. et al. Sexually selective cognition: beauty captures the mind of the beholder. Journal of Personality and Social Psychology, [S.1.], v. 85, n. 6, p. 1107-1120, 2003.

MARTINS, F. C. de O.; SASSI JÚNIOR, E. A comorbidade entre transtornos alimentares e de personalidade e suas implicações clínicas. Revista de Psiquiatria Clínica, [S.1.], v. 31, n. 4, p. 161-163, 2004.

MORGAN, C. M. Boas novas para os pesquisadores de transtornos alimentares no Brasil. Revista Brasileira de Psiquiatria, [S.1.], v. 27, n. 3, p. 255-256, set. 2005.

MOSCOVICI, S. A representação social da psicanálise. Tradução de Álvaro Cabral. Rio de Janeiro: J. Zahar, 1978.

MOSCOVICI, S. Representações sociais: investigações em Psicologia Social. Petrópolis, RJ: Vozes, 2003.

NIEMEYER, F.; KRUSE, M. H. L. Construindo sujeitos anoréxicos: discursos da revista Capricho. Texto \& Contexto - Enfermagem, [S.1.], v. 17, n. 3, p. 457-465, jul./set. 2008.

NÓBREGA, T. P. Agenciamentos do corpo na sociedade contemporânea: uma abordagem estética do conhecimento da educação fisica. Motrivivência, Florianópolis, v. 7, n. 16, p. 15-21, mar. 2001. Disponível em: <https://journal. ufsc.br/index.php/motrivivencia/article/view/4967/5134>. Acesso em: 21 maio 2007.

NOVAES, J. V.; VILHENA, J. De Cinderela a Moura Torta: sobre a relação mulher, beleza e feiúra. Interações, Estudos e Pesquisas Psicológicas, [S.1.], v. 8, n. 15, p. 9-36, jan./jun. 2003.

PASQUALI, L. Instrumentos psicológicos: manual prático de elaboração. Brasília: LabPAM - IBAPP, 1999.

PEREIRA, J. C. R. Análise de dados qualitativos: estratégias metodológicas para as ciências da saúde, humanas e sociais. São Paulo: Edusp, 1999. 
PINZON, V.; NOGUEIRA, F. C. Epidemiologia, curso e evolução de transtornos alimentares. Revista de Psiquiatria Clínica, [S.1.], v. 31, n. 4, p. 158-160, 2004.

QINGXING, P., XUE, W.; JIAYE, Z. The rise and prospects of medical aesthetics. Social Science and Medicine, [S.1.], v. 41, n. 8, p. 1197-1201, 2000.

QUEIROZ, R. S. (Org.). O corpo do brasileiro: estudos de estética e beleza. São Paulo: Senac, 2000.

RODRIGUES, A.; ASSMAR, E. M. L.; JABLONSKI, B. (Org.). Atitudes: conceito, formação e mudança. In: Psicologia Social. 21. ed. Petrópolis, RJ: Vozes, 2002. p. 204-243.

SALZANO, F. T.; CORDÁS, T. A. Hospital dia (HD) para transtornos alimentares: revisão de literatura e primeiras impressões do HD do ambulatório de bulimia e transtornos alimentares do I. Pq. H. CFMUSP (AMBULIM). Revista de Psiquiatria Clínica, [S.1.], v. 30, n. 3, p. 86-94, 2004 a.

SALZANO, F. T.; CORDÁS, T. A. Tratamento farmacológico de transtornos alimentares. Revista de Psiquiatria Clínica, [S.1.], v. 31, n. 4, p. 188-194, 2004 b.

SERRA, G. M. A.; SANTOS, E. M. Saúde e mídia na construção da obesidade e do corpo perfeito. Ciência e Saúde Coletiva, [S.1.], v. 8, n. 3, p. 691-701, 2003.

SOARES NETO, J. F. P. Santé. In: ANDRIEU, B. (Org.). Le dictionnaire du corps em sciences humaines e sociales. Paris: CNRS, 2006. p. 455-457.

SOUTO, S.; FERRO-BUCHER, J. S. N. Práticas indiscriminadas de dietas de emagrecimento e o desenvolvimento de transtornos alimentares. Revista de Nutrição, [S.1.], v. 19, n. 6, p. 693-704, 2006.

SOUZA, A. de F. C. O percurso dos sentidos da beleza através dos séculos: uma análise discursiva. 2004. Dissertação (Mestrado)-Unicamp, Campinas, São Paulo, 2004.

STENZEL, L. M.; GUARESCHI, P. A. A dialética obesidade/magreza: um estudo em representações sociais com adolescentes. Revista de Ciências Humanas, [S.1.], v. 1, n. 1, p. 183-194, maio/ago. 2002.

STRAUB, R. O. Psicologia da saúde. Porto Alegre: Artmed, 2005.

TAVARES, M. da C. G. C. F. Imagem corporal: conceito e desenvolvimento. São Paulo: Manole, 2003. 
TEIXEIRA, S. A. Produção e consumo social da beleza. Horizontes Antropológicos, [S.1.], v. 7, n. 16, p. 189-220, dez. 2001.

TURNER, F. An evolutionary/chaotic theory of beauty and meaning. Journal of Social and Evolutionary Systems, [S.1.], v. 19, n. 2, p. 103-124, 2002.

VALA, J.; MONTEIRO, M. B. (Org.). Psicologia social. Lisboa: Fundação Calouste Gulbekian, 1996.

WEIL, P.; TOMPAKOW, R. O corpo fala: a linguagem silenciosa da comunicação não-verbal. 49. ed. Petrópolis, RJ: Vozes, 1999.

WEINBERG, C.; CORDÁS, T. A.; MUNOZ, P. A. Santa Rosa de Lima: uma santa anoréxica na América Latina? Revista Psiquiátrica do Rio Grande do Sul, [S.1.], v. 27, n. 1, p. 51-56, jan./abr. 2005.

Recebido em: 22 de março de 2012 Aceito em: 03 de julho de 2013 